patients received full resuscitation; even in the $22 \%$ who were classified as receiving limitation of care, $18.8 \%$ were actually transferred out of the intensive care unit (ICU) terminally (left against medical advice) for financial or other reasons. Only $1.6 \%$ of ICU deaths had do-not-resuscitate orders and another $1.6 \%$ had withholding of life support [2]. A second study carried out at four centres in Mumbai [3] revealed that 34\% of deaths had limitation of therapy terminally. Approximately $25 \%$ of these patients were not intubated terminally; $67 \%$ were initially intubated and ventilated but failed to recover and, subsequently, had no further escalation of therapy; and $8 \%$ had withdrawal of therapy [3].

Secondly, apart from the educational, social and cultural differences, the healthcare system in India differs substantially from that in Europe. In Europe, government and national health insurance account for $70 \%$ of total health expenditure [4] compared with $20 \%$ in India, where $80 \%$ of the total healthcare bill is paid by the patient or their relatives [5].

Thirdly, the ethical and legal status of withholding and withdrawal of life-sustaining therapy from critically ill patients in India is ambiguous. Concepts like autonomy and death with dignity have not been explored in any meaningful way by the constitution. Euthanasia and physician-assisted suicide are not legal. Consequently, physicians are often reluctant to proactively limit therapy.

Fourthly, India has less than one hospital bed per 1,000 people and an even lower number of ICU beds [5].

Given the scarcity of resources and growing needs in India, it is the right time for physicians and allied healthcare societies to educate the government and public about the magnitude of the problem, and to start a healthy dialogue in order to reach a constitutional and legal directive with regard to withholding and withdrawal of care in critically ill patients.

\section{A. Singh}

Christian Medical College and Hospital, Ludhiana, India.

\section{STATEMENT OF INTEREST}

None declared.

\section{REFERENCES}

1 Nava S, Sturani C, Hartl S, et al. End-of-life decision-making in respiratory intermediate care units: a European survey. Eur Respir J 2007; 30: 156-164.

2 Mani RK. Limitation of life support in the ICU. Ethical issues relating to end of life care. Indian J Crit Care Med 2003; 7: 112-117.

3 Kapadia F, Singh M, Divatia J, et al. Limitation and withdrawal of intensive therapy at the end of life: practices in intensive care units in Mumbai, India. Crit Care Med 2005; 33: $1272-1275$.

4 World Health Organization. The World Health Report 2002: reducing risks, promoting healthy life. Geneva, World Health Organization, 2002.

5 Ministry of External Affairs, Government of India. Healthcare. http:/ / meaindia.nic.in/indiapublication/healthcare.htm. Date created: June 19, 2004. Date last accessed: July 8, 2007.

DOI: $10.1183 / 09031936.00085707$

\title{
Cardiovascular and metabolic effects of CPAP in obese obstructive sleep apnoea patients
}

\section{To the Editors:}

In a recent issue of the European Respiratory Journal, CoUGHLIN et al. [1] demonstrated that, in Caucasians with untreated obstructive sleep apnoea (OSA), continuous positive airway pressure (CPAP) can improve baroreceptor responsiveness and reduce waking blood pressure within 6 weeks, but that this treatment period was insufficient to modify insulin resistance or change the metabolic profile. This is the first randomised placebo-controlled blinded crossover trial comparing cardiovascular and metabolic outcomes after 6 weeks of therapeutic and sham CPAP in obese symptomatic Caucasians with OSA. We have found similar CPAP effects in obese Japanese OSA patients (table 1). The authors suggested that there is a need to offer multiple modalities of treatment to obese OSA patients if their cardiovascular risk profile is to be successfully modified. We totally agree with their conclusion. However, it may be necessary to address the following unresolved issues.

1) Although the participating OSA subjects were randomised to receive either therapeutic or identical sham CPAP, sham
CPAP may not be the best placebo treatment. Although therapeutic CPAP improves sleep quality in OSA patients, sham CPAP may not always improve sleep quality and daytime function in patients [2]. Healthy individuals without OSA may experience night-time CPAP as a form of torture. However, patients with severe OSA have a good night's sleep with CPAP.

2) The severity of the OSA may influence the effect of CPAP on metabolic outcomes. As shown in table 1, CPAP treatment exerted significant effects on some metabolic variables in very severe OSA (apnoea/hypopnoea index (AHI) $>45$ events $\cdot h^{-1}$ ), but not in moderate-to-severe OSA (AHI $<45$ events $\cdot h^{-1}$ ).

3) There is an effect of sex on metabolic outcomes and sleep apnoeas [3]. Risk factors for metabolic syndrome also differed by sex; in males, age, body mass index (BMI) and OSA (AHI $\geqslant 15$ events $\cdot h^{-1}$ ) were significantly associated with metabolic syndrome, whereas in females, BMI was the only risk factor [4].

4) The effect of short-term withdrawal of CPAP therapy on cardiovascular and metabolic variables may be of interest in 


\begin{tabular}{|c|c|c|c|c|}
\hline \multirow[t]{3}{*}{ TABLE 1} & $\begin{array}{l}\text { uous positive airv } \\
\text { ea (OSA) }\end{array}$ & ssure (CPAP) tre & components of $r$ & lic syndrome in \\
\hline & \multicolumn{2}{|c|}{ Severe OSA ${ }^{\#}$} & \multicolumn{2}{|c|}{ Nonsevere OSA } \\
\hline & Good compliance $^{+}$ & Poor compliance ${ }^{\S}$ & Good compliance ${ }^{+}$ & Poor compliance ${ }^{\S}$ \\
\hline Subjects $n$ & 42 & 20 & 32 & 20 \\
\hline ESS & $15.2 \pm 0.4$ & $14.7 \pm 0.9$ & $11.7 \pm 0.9$ & $11.2 \pm 0.5$ \\
\hline$\Delta \mathrm{ESS}$ & $-11.2 \pm 0.4^{*}$ & $-7.8 \pm 0.9^{*}$ & $-7.2 \pm 0.9^{*}$ & $-4.8 \pm 0.5^{\star}$ \\
\hline \multicolumn{5}{|l|}{ BP $\mathrm{mmHg}$} \\
\hline Systolic & $145.7 \pm 2.0$ & $143.4 \pm 2.1$ & $138.7 \pm 2.0$ & $137.5 \pm 2.4$ \\
\hline Diastolic & $86.1 \pm 1.5$ & $86.7 \pm 1.6$ & $86.7 \pm 1.6$ & $86.7 \pm 1.6$ \\
\hline$\triangle H O M A-I R$ & $-0.2 \pm 0.1$ & $-0.1 \pm 0.1$ & $-0.1 \pm 0.1$ & $0.1 \pm 0.1$ \\
\hline Cholesterol mM & $5.6 \pm 0.1$ & $5.5 \pm 0.1$ & $5.6 \pm 0.1$ & $5.5 \pm 0.1$ \\
\hline$\Delta$ Cholesterol mM & $-0.2 \pm 0.1$ & $-0.1 \pm 0.1$ & $-0.1 \pm 0.1$ & $0.1 \pm 0.1$ \\
\hline Triglycerides mM & $1.9 \pm 0.2$ & $1.8 \pm 0.2$ & $1.9 \pm 0.3$ & $1.9 \pm 0.2$ \\
\hline$\Delta$ Triglycerides $\mathrm{mM}$ & $-0.1 \pm 0.1$ & $0.0 \pm 0.1$ & $-0.1 \pm 0.1$ & $0.1 \pm 0.1$ \\
\hline
\end{tabular}

Data are presented as mean \pm SEM. AHI: apnoea/hypopnoea index; $\Delta$ : change (post-CPAP value minus pre-CPAP value); ESS: Epworth Sleepiness Scale; BP: blood pressure; SBP: systolic BP; FPG: fasting plasma glucose; HOMA: homeostasis model assessment; IR: insulin resistance. ${ }^{\#}:$ AHI $\geqslant 45$ events $\cdot h^{-1} ;{ }^{\bullet}: 45$ events $\cdot h^{-1}>A H I \geqslant 15$ events $\cdot h^{-1}$; $t_{:}>5$ h.night ${ }^{-1}$ CPAP; ${ }^{\text {s. }}<5$ h.night ${ }^{-1}$ CPAP. *: $\mathrm{p}<0.05$ versus pre-CPAP value.

OSA patients. This inverse method may also confirm the randomised placebo-controlled blinded crossover trial results. It was recently reported that 1 week of CPAP withdrawal is associated with a return of OSA and a marked increase in sympathetic activity without concomitant elevation of vascular inflammatory marker levels [5]. Therefore, effects of CPAP treatment and its withdrawal may differ between cardiovascular function and metabolic and inflammatory function as a function of time.

5) The relationships between metabolic variables and systemic inflammation and sympathetic activity are complex [6]. There is a positive correlation between interleukin (IL)- 6 or tumour necrosis factor (TNF)- $\alpha$ plasma levels and BMI. IL- 6 , TNF- $\alpha$ and insulin levels are elevated in sleep apnoea independently of obesity and visceral fat [7, 8]. Conversely, recent data suggest that OSA has no independent association with lipid abnormalities, insulin resistance, and serum leptin and adiponectin levels. On multivariate analysis, obesity was the major determinant of metabolic abnormalities [9].

Furthermore, there is a maladaptive autonomic response of chemoreceptors, reacting to the hypoxia, hypercapnia and acidosis of sleep apnoea in OSA patients. The elevated sympathetic response triggers an inflammatory cascade that results in a myriad of downstream consequences, including insulin resistance, hypertension, diabetes, atherosclerosis and metabolic syndrome. The sympathetic bias and endocrine disturbances may further exacerbate sleep disturbance in a potentially pernicious cycle.
6) Poor compliance with CPAP may considerably affect metabolic outcomes [10]. Unfortunately, compliance with CPAP was generally very low in the population-based sample. Furthermore, the patients with more severe OSA may show greater CPAP use than those with mild-to-moderate OSA. Both compliance with CPAP and patient selection may have affected the results of the current study.

Even though we totally agree with the main results of the study by COUGHLIN et al. [1], changes in cardiovascular and metabolic variables following CPAP treatment may differ. Although more detailed results from a broad-ranging population with OSA are needed, OSA and its related metabolic abnormalities should be treated by means of CPAP and other useful modalities, including statins, angiotensin II receptor blockers, etc.

We believe that continuous positive airway pressure treatment is effective at reducing cardiovascular events through reduced blood pressure, decreased sympathetic activity and reduced systemic inflammation [10]. However, the relative contributions to the reduction in cardiovascular events should be further elucidated in terms of obstructive sleep apnoea severity and the basic mechanisms of metabolic syndrome.

\section{S. Teramoto*, Y. Yamaguchi*, H. Yamamoto*, Y. Hanaoka*,} M. Ishii*, S. Hibi*, T. Ishii*, H. Kume ${ }^{\#}$ and Y. Ouchi* Depts of *Geriatric Medicine, and "Urology, Graduate School of Medicine, University of Tokyo, Tokyo, Japan. 


\section{STATEMENT OF INTEREST}

None declared.

\section{REFERENCES}

1 Coughlin SR, Mawdsley L, Mugarza JA, Wilding JP, Calverley PM. Cardiovascular and metabolic effects of CPAP in obese males with OSA. Eur Respir J 2007; 29: 720-727.

2 Teramoto S, Ohga E, Ouchi Y. Obstructive sleep apnoea. Lancet 1999; 354: 1213-1214.

3 Teramoto S, Kume H, Yamaguchi Y, et al. Improvement of endothelial function with allopurinol may occur in selected patients with OSA: effect of age and sex. Eur Respir J 2007; 29: 216-217.

4 Sasanabe R, Banno K, Otake K, et al. Metabolic syndrome in Japanese patients with obstructive sleep apnoea syndrome. Hypertens Res 2006; 29: 315-322.

5 Phillips CL, Yang Q, Williams A, et al. The effect of shortterm withdrawal from continuous positive airway pressure therapy on sympathetic activity and markers of vascular inflammation in subjects with obstructive sleep apnoea. J Sleep Res 2007; 16: 217-225.
6 Vgontzas AN, Bixler EO, Chrousos GP. Sleep apnoea is a manifestation of the metabolic syndrome. Sleep Med Rev 2005; 9: 211-224.

7 Teramoto S, Yamamoto $H$, Yamaguchi Y, Namba R, Ouchi Y. Obstructive sleep apnoea causes systemic inflammation and metabolic syndrome. Chest 2005; 127 1074-1075.

8 Teramoto S, Yamamoto H, Ouchi Y. Increased C-reactive protein and increased plasma interleukin- 6 may synergistically affect the progression of coronary atherosclerosis in obstructive sleep apnoea syndrome. Circulation 2003; 107: E40-0.

9 Sharma SK, Kumpawat S, Goel A, Banga A, Ramakrishnan L, Chaturvedi P. Obesity, and not obstructive sleep apnoea, is responsible for metabolic abnormalities in a cohort with sleep-disordered breathing. Sleep Med 2007; 8: 12-17.

10 Lindberg E, Berne C, Elmasry A, Hedner J, Janson C. CPAP treatment of a population-based sample - what are the benefits and the treatment compliance? Sleep Med 2006; 7: 553-560. 